\title{
Psychosomatic Disease (PSD) in the Digestive System
}

\author{
Fujio Matsunaga, M. D. \\ The First Medical Department, Hirosaki University \\ School of Medicine, Hirosaki, Japan
}

\section{Introduction}

It is not at all difficult to present cases with psychosomatic disease (PSD), but not easy to study PSD from the viewpoint of clinical statistics to understand how much the psychogenic factors in relation with patient's personality, predisposition to the disease, are playing in the occurrence, progress and/or reccurrence of gastrointestinal diseases.

I dared to study this difficult subject with my own criteria for PSD in a narrow sense by several methods of approach.

\section{Studies in cases with ulcerative colitis}

My interest in PSD began with the studies on ulcerative colitis, which I, as the special speaker, presented at the 55th annual meeting of the Japanese Society of Internal Medicine. At that time projective method (sentence completion test - SCT-, picture frustration test - PFT - and Rorschach test) was used for the analysis of personality. Patients with ulcerative colitis showed immature, dependent and psyche-poor personality in characteristics. But, it was not pathognomonic for the disease, and I added to my conclusion that such personality was also common in patients with other colonic diseases.

\section{Material and method of the present study}

For such study as clinical statistics of PSD physician's subjectivity must be avoided as much as possible, therefore, necessitating criteria with objective findings for PSD in a narrow sense. According to my opinion PSD in a narrow sense should be limited to organic diseases, but to go along with the chairman Professor Ikemi's opinion, patients with organ neurosis and the related functional disorders were also chosen for the objects of this study. The objects were all in-patients. Besides the time consuming interview, three of the personality tests with questionaires were performed in each patient. 


\section{Conflicts as possible cause of stress obtained by interview}

Interview with each patient was made by the same physician Dr. K. Kawakami in our department. The discovery rate of conflicts was as follows in various digestive diseases.

The highest rate was seen in aerophagia with $75 \%$, followed by duodenal ulcer, gastric antrospasm, splanchnoptosis and irritable colon syndrome in order, all approximately with $65 \%$. In gastric ulcer the rate was $50 \%$ and in chronic colitis of various forms it was lowest, $33 \%$. The conflicts were related to social problems $(46 \%)$, familial problems $(37 \%)$, environmental problems $(9 \%)$ and anxiety for diseases, particularly for cancer (8\%).

Patients with diseases of the stomach or duodenum, particularly with the ulcers, showed a high incidence of conflicts related to social problems. This was especially true with the brain workers in a high social position. In contrast, patients with chronic diseases showed a relatively high incidence of conflicts related to familial problems.

The discovery rate was $56 \%$ in the over-all organic diseases whereas it was $69 \%$ in the group of functional disorders. The rate difference between the two was insignificant as described $x^{2}=1.63$.

\section{Personality test with questionaires and the results}

Besides the Yatabe-Guilford test, which is widely used in this country, Cornell Medical Index (CMI) and Minnesota Multiphasic Personality Inventory (MMPI) were performed, and the results were compared. 38 student nurses were studied as controls. $1 / 5$ to $1 / 7$ of supposedly normal controls turned out as neurotic in each test. Inclusion of these with neurosis suspect by my criteria which will be discussed later made the incidence of neurosis quite high: Yatabe-Guilford (38\%), CMI (41\%) and MMPI (48\%). Results of the three tests showed the same tendency in general, but unfortunately not necessarily so in individual cases.

Yatabe-Guilford test: Left-lower type seemed to indicate neurosis and right-sided type neurosis suspect. No definite conclusion could be made by the comparison of the average value in each gastrointestinal disease, but leftlower type was quite typical in aerophagia.

CMI: Grade 4 and grade 3 in Fukamachi's classification were considered to indicate neurosis and neurosis suspect respectively. Average value in the controls was found in grade 2 whereas that in both organic diseases and functional disorders was in grade 3 .

MMPI: Definite elevation of F on adequacy scale with biphasic elevation at $\mathrm{Hs} \cdot \mathrm{D} \cdot \mathrm{Hy}$ and $\mathrm{Pt}$ on clinical scale was considered to indicate a typical 
neurosis. Biphasic elevation without elevation of $\mathrm{F}$ was considered to indicate a neurosis suspect. There was no definite sex characteristics in the values in the controls. However, in the females with neurosis or nuerosis suspect prominent $\mathrm{F}$ was seen, and in the male patients peaks of D, Mf and Sc were prominent. In patients with duodenal ulcer or irritable colon syndrome, on the other hand, $F$ was seen within the normal limits but a high pak at the neurotic triad on clinical scale and a slight elevation at the psychotic phase were observed.

In the total results of the three tests a striking tendency of neurotic personality was seen in duodenal ulcer among the organic diseases and such was seen in the functional disorders, that is irritable colon syndrome, aerophagia and gastric antrospasm. Because of the occasional disagreement among the results of the three tests in individual cases, an average value in each disease group would flatten the characteristic peaks, thus showing itself inadequate for general evaluation (Fig. 1).

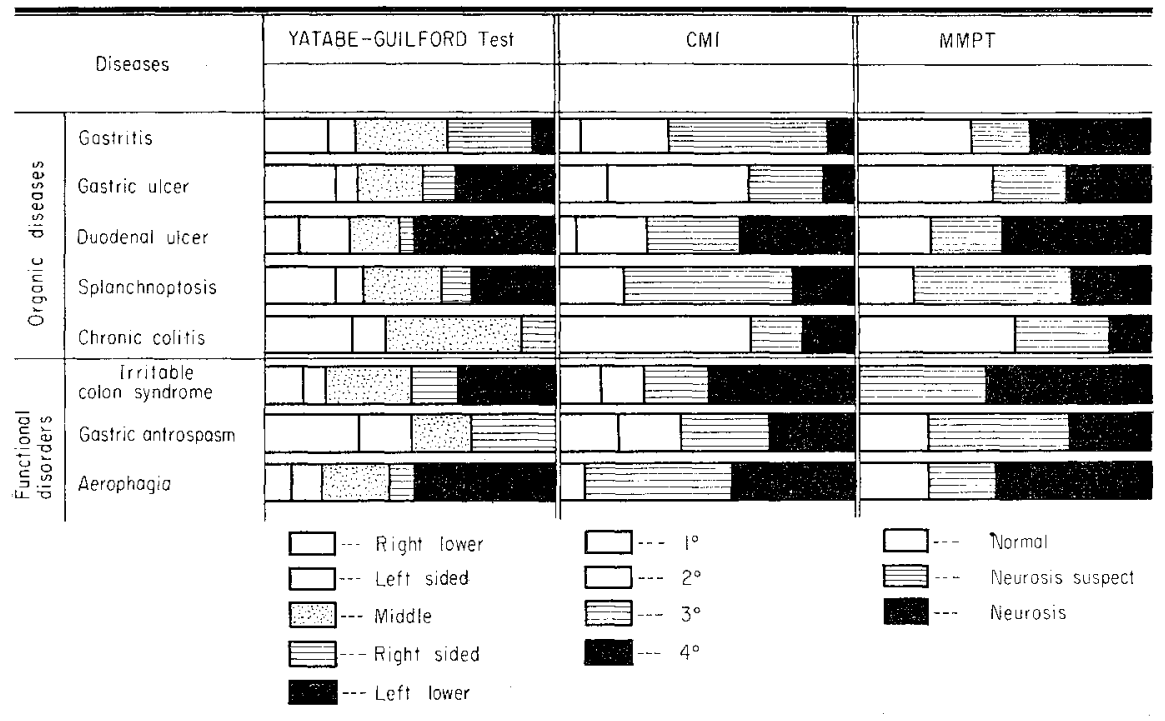

Fig. 1. Results of the questionnaire tests in various diseases.

\section{Criteria for PSD in a narrow sense}

Based on the data above, I have postulated criteria for PSD in a narrow sense. Fundamentally speaking, PSD in a broad sense would involve almost all of the diseases in internal medicine. Definition of PSD in a narrow sense, on the other hand, has not been agreed upon among the authors.

It is clinically practical and convenient to distinguish PSD from neurosis 
and othe related diseases by limiting the former to organic diseases. However, as Professor Ikemi states, the diseases believed to be functional at the present day might become considered organic with better diagnostic tools in the future. Therefore, organ neurosis and the related functional disorders were included, as exceptions, in the PSD in a narrow sense in this study, although a PSD should be an organic disease as a rule (first criterion).

The second criterion for PSD is that the physician must find conflicts as the cause of chronic stress by interviewing with the patient. This criterion, however, is defective in the following three points; 1) An apparent conflict may not be a causative factor in the particular disease; 2) Interview may fail in finding the conflicts; 3 ) The latent conflicts, which cannot be brought out otherwise but by the psychoanalysis or other highly specialized methods, would be more significant in etiology than the apparent conflicts. However, the above considerations would involve the field beyond the clinical practice as internists, and the present study neglected the considerations purposely.

The third criterion is that two out of the three personality tests must show indications for neurotic personality or at least for such tendency.

Clinical data were compared in the PSD, where the above criteria were satisfied, and in the non-PSD. In the non-PSD eosinophile disappearance rate in Thorn's ACTH test was 52.4\% on average, which is within the normal

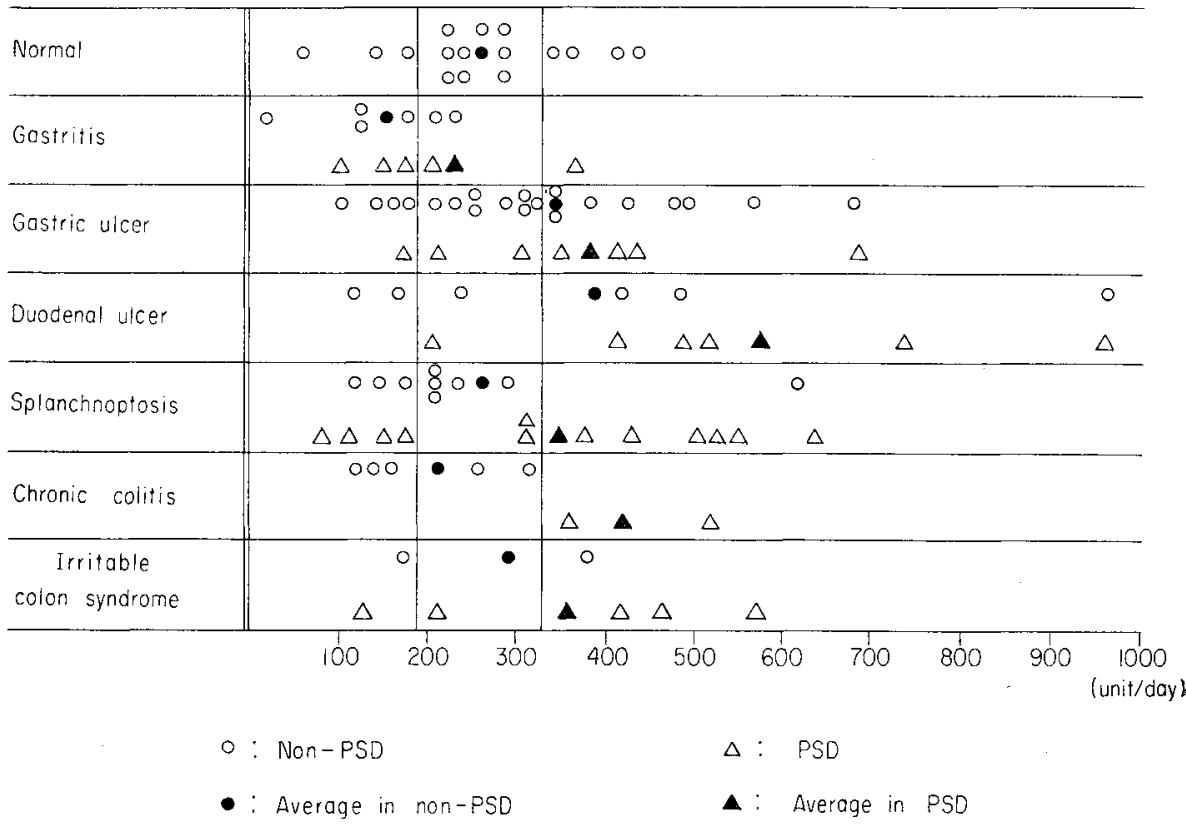

Fig. 2. Uropepsin in PSD and non-PSD. 
range, while it was only $36.9 \%$ in the PSD. 24 hours urinary excretion of 17 KS was $5.31 \mathrm{mg}$. on average in the non-PSD while it was $6.88 \mathrm{mg}$. in the PSD. Uropepsin value (by Sircus) was various in different diseases. In the group of the same disease, however, it was higher in the PSD than in the non-PSD without exception. In the PSD, the uropepsin value was highest in duodenal ulcer and, except in chronic gastritis, all showed a higher average value than the controls (Fig. 2).

\section{Autonomic nervous system and PSD}

When the responses of autonomic nervous system to the mecholyl test were classified to $\mathrm{S}, \mathrm{N}$, and $\mathrm{P}$ types according to Okinaka's criteria. $\mathrm{S}$ type

\begin{tabular}{|c|c|c|c|c|c|c|c|c|}
\hline \multicolumn{2}{|c|}{$\begin{array}{l}\text { Response } \\
\text { Type } \\
\end{array}$} & Cases & $\%$ & 0 & 20 & 40 & 60 & $80 \%$ \\
\hline \multirow{3}{*}{ 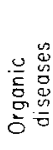 } & S & 4 & 4 & $\square$ & & & & \\
\hline & $N$ & 44 & 43 & & & & & \\
\hline & P & 54 & 53 & & & & & \\
\hline \multirow{3}{*}{ 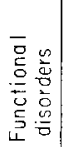 } & s & 3 & 13 & + & & & & \\
\hline & N & 5 & 23 & & 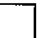 & & & \\
\hline & P & 15 & 65 & & & & & \\
\hline
\end{tabular}

\begin{tabular}{|c|c|c|c|c|c|c|c|c|}
\hline \multicolumn{2}{|c|}{$\begin{array}{c}\text { Response } \\
\text { Type }\end{array}$} & Coses & $\%$ & 0 & 20 & $4 \rho$ & 60 & $80 \%$ \\
\hline \multirow{3}{*}{ 吕 } & S & 6 & 9 & + & & & & \\
\hline & $N$ & 15 & 22 & & & & & \\
\hline & $P$ & 47 & 69 & & & & & \\
\hline \multirow{3}{*}{$\begin{array}{l}0 \\
0 \\
0 \\
1 \\
c \\
0 \\
z\end{array}$} & S & 1 & 2 & $\square$ & & & & \\
\hline & $N$ & 34 & 60 & & & & & \\
\hline & $P$ & 22 & 38 & & & & & \\
\hline
\end{tabular}

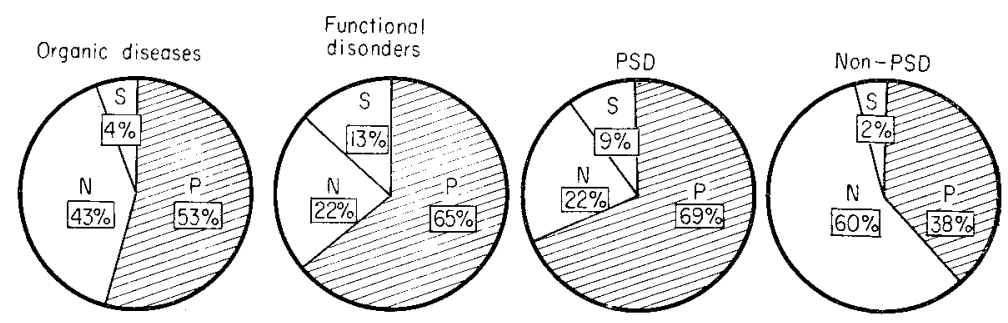

Fig. 3. Results of the mecholyl test. 
was rare in general in patients with gastrointestinal diseases. In the group of organic diseases there was no clear difference in the frequency of $P$ and $\mathrm{N}$ types while in the group of functional disorders $\mathrm{P}$ type came up to $65 \%$ of the cases.

Reconstruction of the above data showed that $69 \%$ of the PSD patients belonged to $P$ type while it was so in only $38 \%$ in the non-PSD patients. Therefore, it is evident and significant that patients with PSD belong more to $\mathrm{P}$ type, that is a vagotonic state (Fig. 3).

\section{Effect of drug therapy on psychogenic symptoms}

Effect of chlordiazepoxide was studied in 50 cases with gastrointestinal diseases, and the effectiveness was seen only in $50 \%$ of all. Among the PSD, however, the effect on symptoms appreared to be much greater.

Insidon was given to another group of patients, and a similar effectiveness was seen. This drug was particularly effective for psychogenic symptoms.

Besides the tranquilizers the psychostimulants will be helpful in easing off the psychogenic stress in the PSD patients. Prescription of these drugs to patients with gastrointestinal disease complicated by PSD is recommended along with the psychological methods of treatment for the relief of the symptoms and for the mental hygiene of the patient. Thus it may be concluded that the drug therapy is effective and significant in the treatment of PSD.

\section{Summary and conclusion}

In order to study psychosomatic diseases from the view-point of clinical statistics, I have postulated criteria for PSD in a narrow sense.

Although my criteria are not the last decisive ones, and there are many border line cases, an apparent difference in the tests studied between PSD and non-PSD by my definition, and between different diseases was still found. Hormonal abnormality in the pituitary-adrenal system, observed by Thorn's test, 17-KS urinary excretion and uropepsin value, and vagotonia, observed by the results of mecholyl test, all favor in distinguishing PSD from non-PSD. Therefore, it may be said that studies as this according to the postulated criteria are significant as well as practical in the investigation of PSD in general, particularly in understanding the characteristics of somatic responses in the disease.

Frequency of PSD in the various clinical diagnoses is as follows: PSD $15 \%$ and PSD suspect $27 \%$ in the group of organic diseases, and $38 \%$ and $31 \%$ respectively in the group of functional disorders. Duodenal ulcer in the former group is close to the latter group in the frequency of PSD and distinguishes 
itself from gastric ulcer. PSD in splanchnoptosis is no more frequent than that in gastritis and gastric ulcer, but PSD suspect was seen in more than half of the patients with the diagnosis. In the latter group, PSD in irritable colon syndrome, aerophagia and gastric antrospasm were 43, 33 and 33\% respectively, and PSD suspect, 21, 42 and $34 \%$ (Fig. 4).

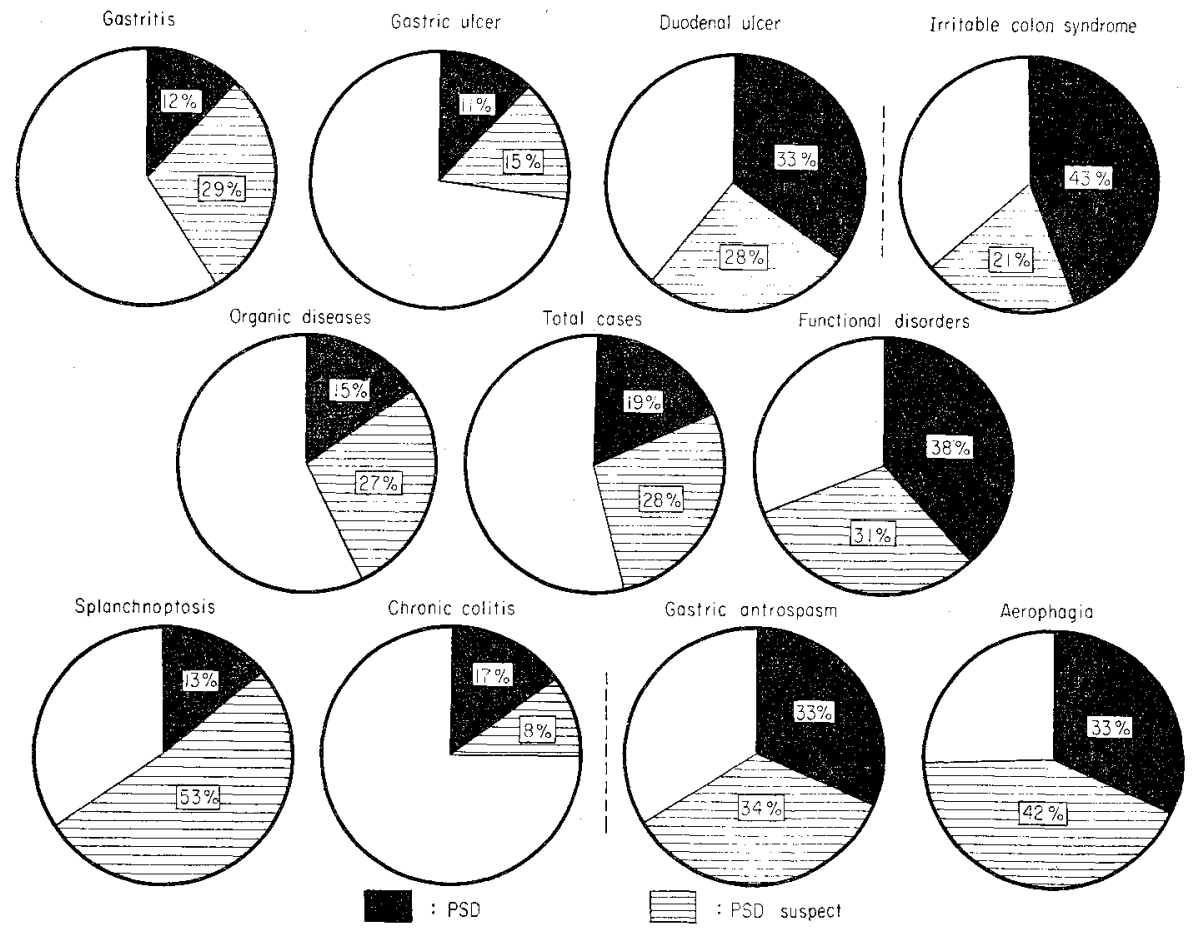

Fig. 4. Frequency of PSD in the various clinical diagnoses.

From the data above one can see that not only in the functional disorders but in the organic diseases also the therapy for the somatic changes alone would not be sufficient. This is particularly true with duodenal ulcer and splanchnoptosis, and has to be considered at such occasions as before surgical procedures.

No specific mention was made on therapy, but it should be done as follows. The first step should be directed to the treatment of the organic disease and then for the psychic stability. As a good human relationship between the physician and the patient is established, professional assistance should be directed to the analysis and resolution of the basic problem, psychogenic factors. This may be a generally accepted approach of an internist in this country and has been our usual practice. 